\title{
Modelo de estimación de matrices y flujo en rutas ruta usando densidad de flujos en arcos
}

\author{
Louis de Grange and Felipe González \\ Escuela de Industrias, Universidad Diego Portales, Santiago, Chile \\ Shlomo Bekhor \\ Faculty of Civil and Environmental Engineering Technion - Israel Institute of Technology \\ Haifa 32000, Israel.
}

\section{RESUMEN}

En este trabajo presentamos un modelo que permite estimar, simultáneamente, flujos en rutas y matrices de viajes en redes de transporte congestionadas, usando información sobre la densidad vehicular en los arcos de la red, bajo un enfoque macroscópico. La ventaja de usar densidades en lugar de flujos es que evita errores que se producen al relacionar flujo y costo en cada arco cuando la congestión es alta. Además, la metodología permite estimar matrices y flujos en rutas usando una imagen de la red (e.g. fotografía que identifique el número de autos en cada arco). El modelo se basa en formular un problema de optimización de máxima entropía sujeto a restricciones lineales que se obtienen de los datos de densidad vehicular en los arcos de la red.

\section{INTRODUCCIÓN}

En este trabajo presentamos un modelo analítico que permite obtener, de manera simultánea, estimadores de flujos en rutas y de matrices de viajes, en un contexto de modelación estática de redes transporte congestionadas (consideramos un enfoque de equilibrio estático). El modelo utiliza, además de la información de costos sobre los arcos, información sobre la densidad de vehículos en cada arco de la red. Esta información puede obtenerse directamente de una imagen estática de la red vial (e.g. fotografía que identifique el número de autos sobre cada arco de la red en un determinado instante de tiempo), suponiendo que se conoce la relación fundamental del tráfico para cada arco (relación entre velocidad, flujo y densidad), en un contexto macroscópico.

La principal ventaja de usar densidades es que permite capturar de mejor manera la relación entre flujo y costo (tiempo de viaje o velocidad) en cada arco de la red. Los modelos clásicos de estimación de matrices con conteos de flujo en presencia de congestión (ver referencias en la sección 2), consideran normalmente una relación creciente y convexa entre costo y flujo en cada arco de la red, cuando en realidad dicha relación presenta una forma muy distinta para casos de alta congestión, tal como se aprecia en la ecuación fundamental del tráfico que relaciona flujo, velocidad y densidad. Luego, al usar densidad directamente en lugar de flujo, podemos estimar costos para cada arco de mejor forma. 
Otra ventaja que presenta el enfoque macroscópico que proponemos es que no es necesario contar flujos vehiculares en cada arco, sino que basta con conocer el número de autos en cada arco de la red para un determinado instante de tiempo (densidad). Sin embargo, ello requiere conocer una relación entre velocidad, flujo y densidad para cada arco. Esta información se puede obtener fácilmente mediante la instalación de "loop detectors" en diferentes arcos de la red vial.

\section{REVISIÓN DE LA LITERATURA}

Tradicionalmente se han considerado 2 enfoques para construir matrices origen-destino de viajes a partir de conteos de tráfico.

El primer enfoque corresponde a modelos de máxima entropía, que consisten en estimar, a partir de un determinado criterio de información (máxima entropía, siguiendo a Wilson (1970))), la matriz de viajes más probable que sea consistente con los flujos observados (Van Zuylen and Willumsen, 1980). Este es un modelo de programación matemática que intenta determinar la matriz de viajes más idónea que sea consistente con la información contenida en los datos del volumen de tráfico en los arcos de la red, a la vez que maximiza la entropía o usa una función objetivo basada en la mínima información con respecto a la matriz de viajes previa. Este enfoque es estático, y no considera el efecto de la congestión y tiempos de viaje sobre la matriz estimada, por lo que existe una proporcionalidad lineal entre flujos y viajes de la matriz que podría no ser correcta. Para ello, normalmente se plantea un problema de optimización sujeto a restricciones de conservación de flujos sobre una red vial.

Otros investigadores, como Hall et al. (1980), Van Zuylen (1981), Van Vliet and Willumsen (1981), Willumsen (1981, 1982; 1984), Bell (1983), Fisk and Boyce (1983), Nguyen (1984), y Hammerslag and Immers (1988), han realizado tests o han propuesto mejoras y refinamientos para este tipo de modelos.

En general, e independiente del enfoque de modelación usado, los modelos para estimar matrices de viajes usando información de flujos en arcos utilizan la siguiente ecuación que relaciona viajes entre zonas con flujos en arcos:

$$
f_{a}=\sum_{i j} T_{i j} p_{i j}^{a}, \quad 0 \leq p_{i j}^{a} \leq 1
$$

La variable $f_{a}$ es el flujo en el arco a, y debe ser igual a la suma de todos los viajes de la red entre los distintos pares origen-destino $(\mathrm{i}, \mathrm{j})$ que utilizan dicho arco. La variable $T_{i j}$ son los viajes entre la zona de origen i y la zona de destino j. j La variable $p_{i j}^{a}$ es la proporción de viajes entre el par $(\mathrm{i}, \mathrm{j})$ que utilizan el arco a. El valor de la variable $p_{i j}^{a}$ puede ser estimado a partir de diferentes criterios de asignación de tráfico (Farhangian and LeBlanc, 1982; Iida 
et al., 1994).

Por otra parte, un modelo de estimación de flujos en rutas puede presentarse como un proceso conjunto de modelación simultánea de elección de ruta y la estimación de una matriz de viajes. En el trabajo de Bell et al. (1997) se presenta un modelo de asignación estocástica (del tipo Logit) para redes congestionadas que permite estimar las probabilidades de escoger distintas rutas disponibles para un determinado par origen-destino de viajes. Se considera explícitamente la relación flujo-demora de los arcos de las distintas rutas.

Modelos de estimación de flujos en rutas también se presentan en Chen et al. (2005) para estimar matrices de viajes en redes pequeñas de autopistas. El estudio usa conteos de tráfico para estimar los flujos. En otro estudio de Chen et al. (2006) se estiman flujos en rutas para flujos de turistas en ciudades pequeñas.

Un enfoque alternativo para estimar matrices de viajes corresponde al uso de técnicas estadísticas, como mínimos cuadrados generales o máxima verosimilitud. Estas técnicas también se basan en información de flujos en arcos para estimar las matrices de viajes. El enfoque de Máxima Verosimilitud busca encontrar aquella distribución de viajes que sea más consistente con los flujos observados, para una determinada función de densidad. Por otra parte, el método de mínimos cuadrados generales busca estimar una matriz cuya asignación sobre la red vial minimice las diferencias entre los flujos observados y modelados; cada arco de la red puede tener un peso diferente.

Respecto a la relación flujo-velocidad-densidad bajo un enfoque macroscópico podemos decir que normalmente, los flujos de tráfico no son uniformes, sino que varían en el espacio y en el tiempo, lo que representa un fenómeno difícil de describir. Sin embargo, su comportamiento tradicionalmente se explica relacionando tres variables: volumen de flujo, velocidad, y densidad (o concentración).

La velocidad media (v) se define como velocidad promedio de varios vehículos que pasan por una ubicación específica. El flujo f (o volumen) se define como el número de vehículos que cruzan una sección específica dentro de un período de tiempo determinado. Densidad o concentración (D) se define como el número de vehículos que ocupa una sección o tramo particular de la calle, carretera o camino.

Para carreteras o caminos con varias pistas, el flujo se expresa por unidad de tiempo por cada pista de la carretera, pero debido a la condición de tráfico mixto, el flujo se expresa como vehículos por unidad de tiempo por el número total de pistas existentes (Arasan y Krishnamurthy, 2008). Lo mismo ocurre con la densidad, que también se expresa en términos de ancho total de la carretera.

Una buena revisión de este tipo de modelos se puede consultar en Wang et al. (2009). El 
primero y probablemente más simple modelo macroscópico de flujo de tráfico, fue propuesto por Greenshield (1935). Supone que, bajo condiciones de flujo ininterrumpido, la velocidad y la densidad están relacionados linealmente. Este modelo es considerado como la herramienta de gran alcance en el campo de la modelización del flujo de tráfico debido a su sencillez y la bondad de ajuste razonable. Sin embargo, su aceptación no es universal, ya que no presenta un buen ajuste estadístico cuando hay bajos niveles de congestión.

May and Keller (1967) proponen la siguiente relación entre velocidad y densidad de flujo.

$$
v=v_{\min }+\left(v_{\max }-v_{\min }\right)\left(1-\left(\frac{D}{D_{j}}\right)^{\alpha}\right)^{\gamma}
$$

donde $v_{\min }$ corresponde a la velocidad mínima; $v_{\max }$ corresponde a la velocidad máxima; $\mathrm{y}$ $\alpha, \gamma$ son parámetros a calibrar.

La decisión final del modelo a utilizar dependerá principalmente de la bondad de ajuste estadístico de cada uno respecto de los datos disponibles. Como veremos más adelante, nosotros optamos por el modelo de May and Keller (1967).

\section{MODELO DE ESTIMACIÓN SIMULTÁNEA DE FLUJOS EN RUTAS Y MATRIZ DE VIAJES}

Consideremos que conocemos (o podemos estimar adecuadamente) la densidad vehicular $\left(d_{a}\right)$ en un determinado arco a $\in \mathrm{A}$, donde $\mathrm{A}$ es el conjunto de arcos de la red vial. La densidad la definimos como el número de vehículos existentes en un arco durante un determinado instante de tiempo. Por ejemplo, si tomamos una fotografía a la red vial de una ciudad, podríamos estimar el número de vehículos en cada calle de la ciudad, también la longitud de cada calle, y con ello estimar la densidad (número de autos por kilómetro) para cada calle en el instante de tiempo en que se tomó la fotografía. Luego, identificando el número de vehículos na en el arco a, y la longitud la de dicho arco, la densidad la estimamos como $d_{a}=\left(\frac{n_{a}}{l_{a}}\right)$.

Por otra parte, a partir de la ecuación fundamental del tráfico podemos relacionar el flujo del arco a $\left(f_{a}\right)$ con su velocidad $\left(v_{a}\right)$ y su densidad: $f_{a}=v_{a} \cdot d_{a}$. Por lo tanto, si podemos estimar la densidad del arco a (denominada $d_{a}^{*}$ ), podremos estimar directamente la velocidad $v_{a}^{*}=v_{a}\left(d_{a}^{*}\right)$ y por lo tanto también estimar el flujo $f_{a}^{*}=v_{a}^{*} \cdot d_{a}^{*}$.

Usando los flujos estimados para los arcos de la red $\left(f_{a}^{*}\right)$, y definiendo mediante algún criterio (Bekhor et al., 2006) el posible conjunto de rutas alternativas utilizadas (choice-set) entre los pares $\mathrm{O}-\mathrm{D}$ de la red $(\mathrm{Rw})$, es posible resolver el siguiente problema de optimización 
entrópico (Rossi et al, 1989; Janson, 1993, Bar-Gera, 2006):

$$
\begin{array}{ll}
\min & Z_{2}=\sum_{w} T_{w}\left(\ln T_{w}-1\right)+\frac{1}{\psi}\left(\sum_{r \in R_{w}} \sum_{w} h_{w}^{r}\left(\ln h_{w}^{r}-1\right)-\sum_{w} T_{w}\left(\ln T_{w}-1\right)\right) \\
\text { s.a.: } & \sum_{r \in R_{w}} h_{w}^{r}=T_{w}, \quad \forall w \quad\left(\mu_{w}\right) \\
& \sum_{w} T_{w}=T, \quad(\Phi) \\
& \sum_{\substack{r \in R_{w} \\
r \supseteq a}} h_{w}^{r}=f_{a}^{*}, \quad \forall a \quad\left(\beta_{a}\right) \\
& h_{w}^{r} \geq 0
\end{array}
$$

donde Tw es la demanda entre el par w, que es una variable de diseño del problema (en el problema (3) el total de viajes $\mathrm{T}$ es exógeno). En este modelo estamos asumiendo que conocemos el total de viajes T. Considerando una situación "steady-state", el total de viajes en la red durante un determinado instante de tiempo, en unas situación de régimen, puede estimarse simplemente como $T=\varphi \sum_{a \in A} n_{a}$, donde na se conocía previamente (e.g. mediante la fotografía satelital) y $\varphi$ es un parámetro positivo.

Derivando el lagrangeano del problema (3) con respecto a $h_{w}^{r}$ se obtiene:

$$
\begin{aligned}
& h_{w}^{r}=T_{w} \frac{\exp \left(\psi \sum_{a \subseteq R_{w}} \beta_{a}\right)}{\sum_{r \in R_{w}} \exp \left(\psi \sum_{a \subseteq R_{w}} \beta_{a}\right)}, \quad \forall r, w \\
& T_{w}=T \frac{\exp \left(L_{w}\right)}{\sum_{w} \exp \left(L_{w}\right)}, \quad \forall w, \quad L_{w}=\frac{1}{\psi} \ln \sum_{r \in R_{w}} \exp \left(\psi \sum_{a \subseteq R_{w}} \beta_{a}\right)
\end{aligned}
$$

La expresión (5) permite obtener una matriz de viajes consistente con las densidades estimadas para cada arco de la red.

Si asumimos que el conjunto de rutas alternativas entre cada par O-D $\left(R_{w}\right)$ es conocido, podemos plantear la siguiente relación:

$$
\frac{\exp \left(\psi \sum_{a \subseteq R_{w}} \beta_{a}\right)}{\sum_{r \in R_{w}} \exp \left(\psi \sum_{a \subseteq R_{w}} \beta_{a}\right)}=\frac{\exp \left(\theta C_{w}^{r}\right)}{\sum_{r \in R_{w}} \exp \left(\theta C_{w}^{r}\right)}, \quad \forall r, w
$$




$$
\frac{\exp \left(\psi \sum_{a \subseteq R_{w}} \beta_{a}\right)}{\sum_{r \in R_{w}} \exp \left(\psi \sum_{a \subseteq R_{w}} \beta_{a}\right)}=\frac{\exp \left(\sum_{k} \theta_{k} \sum_{a} \delta_{a}^{r, w} x_{a, k}\right)}{\sum_{r^{\prime} \in R_{w}} \exp \left(\sum_{k} \theta_{k} \sum_{a} \delta_{a}^{r^{\prime}, w} x_{a, k}\right)}, \quad \forall r, w
$$

La consistencia entre (6) y (7) se ilustra usando el siguiente ejemplo analítico basado en la red de la figura 2. Las rutas y los viajes asignados se describen en la tabla 1.

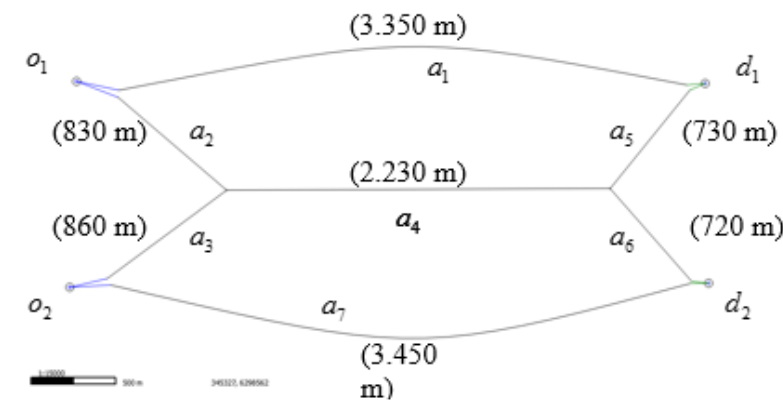

Fig. 2 - Red de Ejemplo

\begin{tabular}{|c|c|l|}
\hline O-D pair $w$ & Trips & \multicolumn{1}{|c|}{ Route link sequences } \\
\hline$o_{1}-d_{1}$ & \multirow{3}{*}{1,500} & R1: $a_{1}$ \\
\cline { 3 - 3 } & & R2: $a_{2}-a_{4}-a_{5}$ \\
\hline$o_{1}-d_{1}$ & & R3: $a_{3}-a_{4}-a_{6}$ \\
\hline$o_{2}-d_{2}$ & \multirow{2}{*}{3,000} & R4: $a_{7}$ \\
\hline$o_{2}-d_{2}$ & & R5: $a_{2}-a_{4}-a_{6}$ \\
\hline$o_{1}-d_{2}$ & 500 & R6: $a_{3}-a_{4}-a_{5}$ \\
\hline$o_{2}-d_{1}$ & 400 & \\
\hline
\end{tabular}

Tabla 1 - Rutas y viajes red de ejemplo

Supongamos la siguiente función velocidad-densidad (May and Keller (1967);

$$
v_{i}=v_{\text {min }}+\left(v_{\max }-v_{\min }\right)\left(1-\left(\frac{D_{i}}{D_{j}}\right)^{\alpha}\right)^{\gamma}
$$

donde:

$v_{t}$ : corresponde a la velocidad promedio en el periodo $t$.

$v_{\min }$ : corresponde a la velocidad mínima. Para la calibración se usó $V_{\min }=0 \mathrm{~km} / \mathrm{hr}$.

$v_{\max }$ : corresponde a la velocidad máxima. Para la calibración se usó $V_{\max }=70 \mathrm{~km} / \mathrm{hr}$.

$D_{t}$ : Densidad promedio en el período $t$.

$D_{j}$ : Jam density, extreme traffic density associated with completely stopped traffic flow.

Para la calibración se usó $D_{j}=150 v e h / \mathrm{km}$.

$\alpha, \gamma:$ Parámetros a calibrar. 
Los valores de los parámetros fueron $\alpha=1.17 ; \gamma=1.28$. Por otra parte, el parámetro $\theta$ del modelo de elección de ruta lo definimos como $\theta=-0.33$ y el parámetro de escala $\psi=1$, para evitar el problema de identificación de parámetros.

La densidad de flujo di en los arcos debe ser consistente, para cada período t, con las funciones de velocidad definidas por (8), pero también con el flujo en rutas $h_{w}^{r}$ y con el flujo en arcos fa. En la Tabla 2 se presentan las densidades en los arcos que aseguran dicha consistencia entre flujos, velocidades (este ejemplo considera un único período de análisis):

\begin{tabular}{|l|c|c|c|c|c|c|c|}
\cline { 2 - 9 } \multicolumn{1}{c|}{} & \multicolumn{7}{c|}{ Arco } \\
\hline Variable & $a_{1}$ & $a_{2}$ & $a_{3}$ & $a_{4}$ & $a_{5}$ & $a_{6}$ & $a_{7}$ \\
\hline Densidad (veh/km) & 16.08 & 15.42 & 25.19 & 73.12 & 13.67 & 27.41 & 35.90 \\
\hline Velocidad (km/hr) & 63.34 & 63.66 & 58.88 & 33.70 & 64.48 & 57.76 & 53.40 \\
\hline Flujo (veh/hr) & 1019 & 981 & 1483 & 2464 & 881 & 1583 & 1917 \\
\hline Largo (km) & 3.35 & 0.83 & 0.86 & 2.23 & 0.73 & 0.72 & 3.45 \\
\hline Tiempo de Viaje (min) & 3.18 & 0.78 & 0.88 & 3.97 & 0.68 & 0.74 & 3.88 \\
\hline
\end{tabular}

Tabla 2 - Datos red de ejemplo

El flujo descrito en la Tabla 2 se introduce en las restricciones del problema de optimización (3). Luego, resolviendo el modelo de optimización entrópico definido en (3) se obtiene los siguientes multiplicadores de Lagrange:

\begin{tabular}{|l|c|c|c|c|c|c|c|}
\hline Link & $a_{1}$ & $a_{2}$ & $a_{3}$ & $a_{4}$ & $a_{5}$ & $a_{6}$ & $a_{7}$ \\
\hline$\beta_{a}$ multipliers & 6.927 & 6.176 & 6.987 & 0.000 & 0.000 & 0.000 & 7.559 \\
\hline
\end{tabular}

Tabla 3 - Multiplicadores de Lagrange

Para las 6 rutas obtenemos:

\begin{tabular}{|l|c|c|}
\hline \multicolumn{1}{|c|}{ Route link sequence } & $\sum_{a \subseteq P_{w}} \beta_{a}$ & $\sum_{a \subseteq P_{w}} t_{a}^{*}$ \\
\hline R1: $a_{1}$ & 6.927 & 3.177 \\
\hline R2: $a_{2}-a_{4}-a_{5}$ & 6.176 & 5.426 \\
\hline R3: $a_{3}-a_{4}-a_{6}$ & 6.987 & 5.588 \\
\hline R4: $a_{4}$ & 7.559 & 3.876 \\
\hline R5: $a_{2}-a_{4}-a_{6}$ & 6.176 & 5.490 \\
\hline R6: $a_{3}-a_{4}-a_{5}$ & 6.987 & 5.524 \\
\hline
\end{tabular}

Tabla 4 - Multiplicadores de Lagrange y tiempos de viaje por ruta

En este caso, dados los valores de la Tabla 1, y considerando el valor $\theta=-0.33$, se cumplen las condiciones de igualdad definidas en (24). Por lo tanto, se confirma la validez del modelo propuesto y en particular la equivalencia expuesta en la ecuación (7). 


\section{CONCLUSIONES}

En este artículo presentamos un modelo que estima flujos en ruta y matrices de viaje para una red de transporte a partir de datos de densidad arcos en lugar de datos de flujos. Este enfoque propuesto tiene la ventaja de incorporar en el análisis, la ecuación fundamental del tráfico en lugar de usar las ecuaciones de flujo demora, que puede ser incorrecto cuando hay mucha congestión.

La formulación propuesta es un modelo de máxima entropía con restricciones de conservación de flujo. Los flujos fueron estimados a partir de las densidades observadas y luego de una relación entre (i) densidad y velocidad y (ii) de flujo, densidad y velocidad.

Nuestra primera conclusión es que el uso de densidades en arco (veh / $\mathrm{km}$ ) en lugar de flujos en arco (veh / h) produce estimaciones satisfactorias de modelos de elección de ruta y matrices de viajes. De hecho, el enfoque basado en densidades en arco proporciona un mejor desempeño, produciendo una estimación insesgada del parámetro poblacional definido en los modelos.

Nuestra segunda conclusión es que la capacidad de predicción del modelo estimado utilizando densidades es superior al modelo estimado usando directamente datos de flujo en arco. Nuestra tercera conclusión es que el enfoque propuesto se puede extender fácilmente a la estimación de matrices de viaje a partir de densidades observadas para redes reales.

Por último, una extensión interesante para futuras investigaciones sería aplicar el enfoque en redes de gran tamaño.

\section{REFERENCIAS}

Arasan, V.T. and Krishnamurthy, K. (2008). Effect of Traffic Volume on PCU of Vehicles under Heterogeneous Traffic Conditions. Road and Transport Research Journal, ARRB, 17, 32-49.

Bar-Gera, H. (2006). Primal method for determining the most likely route flows in large road networks. Transportation Science, 40, 269-286.

Bekhor, S., Ben-Akiva, M. E. and Ramming, S. (2006). Evaluation of choice set generation algorithms. Annals of Operations Research, 144, 235-247.

Bell, M.G.H; Shield, C.M.; Busch, F. and Kruse, G. (1997). A stochastic user equilibrium path flow estimator. Transportation Research, 5C, 197-210.

Chen, A.; Chootinan, P.; Laabs, W. and Recker, W. (2006). Modeling Network Traffic for Planning Applications in a Small Community. Journal of Urban Planning and Development, 132, 156-159. 
Fisk, C.S. and Boyce, D.E. (1983). A note on trip matrix estimation from link traffic count data. Transportation Research, 17B, 245-250.

Hall, M. D.; Van Vliet, D. and Willumsen, L. G. (1980). SATURN: A simulation assignment model for the evaluation of traffic management schemes. Traffic Engineering and Control, $21,168-176$.

Hammerslag, R. and B. Immers (1988). Estimation of trip matrices: shortcomings and possibilities for improvement. Transportation research Record, 1203, 27-39.

Janson, B. (1993). Most likely origin-destination link uses from equilibrium assignment Transportation Research, 27B, 333-350.

May, A. D. and Keller, H. E. (1967). A deterministic queueing model. Transportation Research, 1, 117-128.

Nguyen, S. (1984), Transportation Planning Models, Edited by M. Florian, Elsevier Science Publishers, Amsterdam, chapter Estimating origin-destination matrices from observed flows, 363-380.

Rossi, T.; McNeil, S. and Hendrickson, C. (1989). Entropy model for consistent impact-fee assessment. Journal of Urban Planning and Development, 115, 51-63.

Van Vliet, D. and Willumsen, L.G. (1981). Validation of the ME2 model for estimating trip matrices from traffic counts Proceedings of The Eights International Symposium on Transportation and Traffic Theory, June 1981.

Van Zuylen, H.J. (1981). Some improvements on the estimation of an origin-destination matrix from traffic counts. Proceedings of the 8th International Symposium on Transportation and Traffic Theory, Toronto University, June 1981.

Van Zuylen, J. H. and Willumsen L. G. (1980). The most likely trip matrix estimated from traffic counts. Transportation Research, 14B, 281-293.

Wilson A. G. (1970). Entropy in Urban and Regional Modeling, Pion, London.

Willumsen, L. G. (1981). Simplified transport models based on traffic counts. Transportation, 10, 257-278. 